\title{
Reductive dechlorination of 1,2-dichloroethane in the presence of chloroethenes and 1,2-dichloropropane as co-contaminants
}

\author{
Peng Peng ${ }^{1}$ (D) $\cdot$ Uwe Schneidewind ${ }^{2} \cdot$ Pieter Jan Haest ${ }^{3,4} \cdot$ Tom N. P. Bosma $^{1} \cdot$ Anthony S. Danko $^{5} \cdot$ Hauke Smidt $^{1}$. \\ Siavash Atashgahi ${ }^{1}$
}

Received: 26 March 2019 / Revised: 12 June 2019 / Accepted: 14 June 2019 / Published online: 28 June 2019

(C) The Author(s) 2019

\begin{abstract}
1,2-Dichloroethane (1,2-DCA) is one of the most abundant manmade chlorinated organic contaminants in the world. Reductive dechlorination of 1,2-DCA by organohalide-respiring bacteria (OHRB) can be impacted by other chlorinated contaminants such as chloroethenes and chloropropanes that can co-exist with 1,2-DCA at contaminated sites. The aim of this study was to evaluate the effect of chloroethenes and 1,2-dichloropropane (1,2-DCP) on 1,2-DCA dechlorination using sediment cultures enriched with 1,2-DCA as the sole chlorinated compound (EA culture) or with 1,2-DCA and tetrachloroethene (PCE) (EB culture), and to model dechlorination kinetics. Both cultures contained Dehalococcoides as most predominated OHRB, and Dehalogenimonas and Geobacter as other known OHRB. In sediment-free enrichments obtained from the EA and EB cultures, dechlorination of 1,2-DCA was inhibited in the presence of the same concentrations of either PCE, vinyl chloride (VC), or 1,2-DCP; however, concurrent dechlorination of dual chlorinated compounds was achieved. In contrast, 1,2-DCA dechlorination completely ceased in the presence of $c i$-dichloroethene ( $\mathrm{CDCE}$ ) and only occurred after $\mathrm{cDCE}$ was fully dechlorinated. In turn, 1,2-DCA did not affect dechlorination of PCE, cDCE, VC, and 1,2-DCP. In sediment-free enrichments obtained from the EA culture, Dehalogenimonas 16S rRNA gene copy numbers decreased 1-3 orders of magnitude likely due to an inhibitory effect of chloroethenes. Dechlorination with and without competitive inhibition fit Michaelis-Menten kinetics and confirmed the inhibitory effect of chloroethenes and 1,2-DCP on 1,2-DCA dechlorination. This study reinforces that the type of chlorinated substrate drives the selection of specific OHRB, and indicates that removal of chloroethenes and in particular cDCE might be necessary before effective removal of 1,2-DCA at sites contaminated with mixed chlorinated solvents.
\end{abstract}

Keywords 1,2-Dichloroethane $\cdot$ Co-contaminants $\cdot$ Dehalococcoides $\cdot$ Dehalogenimonas $\cdot$ Dechlorination kinetics

Peng Peng and Uwe Schneidewind contributed equally to this work.

Electronic supplementary material The online version of this article (https://doi.org/10.1007/s00253-019-09985-8) contains supplementary material, which is available to authorized users.

Siavash Atashgahi

siavash.atashgahi@wur.nl

1 Laboratory of Microbiology, Wageningen University \& Research, Wageningen, The Netherlands

2 Department of Civil and Environmental Engineering, Western University, London, ON, Canada

3 Advanced Groundwater Techniques (AGT), Aartselaar, Belgium

4 Division of Geology, Department of Earth and Environmental Sciences, KU Leuven, Heverlee, Belgium

5 Centre for Natural Resources and the Environment (CERENA), Department of Mining Engineering, University of Porto (FEUP), Porto, Portugal

\section{Introduction}

Understanding biodegradation bottlenecks has been a major objective in efforts to harness the metabolic potential of microorganisms for bioremediation of sites contaminated with organic pollutants (Atashgahi et al. 2018; Meckenstock et al. 2015; Vandermaesen et al. 2016). An important class of such contaminants comprises chlorinated solvents such as chloroethenes, chloroethanes, and chloropropanes that have adverse effects on human and environmental health (EPA 2018; Weatherill et al. 2018). Organohalide respiration (OHR) is an example of a microbial metabolism that has been successfully harnessed for engineered remediation of sites contaminated with chlorinated solvents (Atashgahi et al. 2017; Edwards 2014; Ellis et al. 2000). This process is mediated by organohalide-respiring bacteria (OHRB) belonging to distinct genera within the phyla Chloroflexi (e.g., 
Dehalococcoides and Dehalogenimonas), Firmicutes (e.g., Dehalobacter and Desulfitobacterium), and Proteobacteria (e.g., Sulfurospirillum and Geobacter) (Atashgahi et al. 2016).

One of the challenges of bioremediation is the presence of mixtures of organohalogens at contaminated sites. During dechlorination of co-mingled organohalogens, bioattenuation of specific chlorinated solvents has been shown to be prone to inhibition due to the inhibitory effect of dechlorination intermediates on OHRB, their reductive dehalogenase enzymes, and their syntrophic partners (Chan et al. 2011; Dillehay et al. 2014; Grostern et al. 2009; Mayer-Blackwell et al. 2016). For instance, 1,1,1-trichloroethane (1,1,1-TCA) was shown to strongly inhibit chloroethene-reductive dehalogenases of Dehalococcoides (Chan et al. 2011). Moreover, long-term exposure to 1,2-dichloroethane (1,2-DCA) was shown to shift the Dehalococcoides community within a microbial consortium from vinyl chloride (VC) reductive dehalogenase gene (vcrA)containing Dehalococcoides to trichloroethene (TCE) reductive dehalogenase gene (tceA)-containing Dehalococcoides, leading to diminished VC transforming ability (Mayer-Blackwell et al. 2016). In turn, kinetic modeling using the same culture revealed that 1,2-DCA dechlorination was strongly inhibited by cisdichloroethene (cDCE), and efficient 1,2-DCA dechlorination occurred only when cDCE was completely dechlorinated to VC (Mayer-Blackwell et al. 2016). In another study, the presence of 1,1,2-trichloroethane (1,1,2-TCA) and 1,2-dichloropropane (1,2-DCP) inhibited 1,2-DCA dechlorination by Dehalogenimonas lykanthroporepellens BL-DC-9 and D. alkenigignens IP3-3 (Dillehay et al. 2014). An improved understanding of such inhibitory effects can aid in designing bioremediation approaches for sites contaminated with a mixture of chloroethenes, chloroethanes, and/or chloropropanes (Dillehay et al. 2014; Field and Sierra-Alvarez 2004; MayerBlackwell et al. 2016).

Different modeling approaches of varying complexity have been developed to understand the reductive dechlorination of chloroethenes with and without competitive inhibition (Chambon et al. 2013). The description of the reaction kinetics varies from first-order (Corapcioglu et al. 2004; Da Silva and Alvarez 2008) to the more elaborate Michaelis-Menten equations (Garant and Lynd 1998; Haston and McCarty 1999) or Monod kinetics if the responsible OHRB can be sufficiently quantified (Yu and Semprini 2004). The latter two kinetic modeling approaches have been applied at lab and field scales to study competitive inhibition (Christ and Abriola 2007; Lai and Becker 2013; Yu et al. 2005), self-inhibition (Haest et al. 2010a), electron donor limitation (Cupples et al. 2004), dechlorination in the presence of multiple bacterial species (Brovelli et al. 2012; Duhamel and Edwards 2006; Haest et al. 2010b), and dechlorination in conjunction with fermentation, sulfate reduction, or methanogenesis (Kouznetsova et al. 2010; Malaguerra et al. 2011). The Michaelis-Menten and Monod kinetic modeling approaches can also be used to study the dechlorination of chloroethanes or chloropropanes; however, examples in literature are rare. Notable exceptions are Mayer-Blackwell et al. (2016) who studied concurrent dechlorination of 1,2-DCA and cDCE, and Colombani et al. (2014) who studied 1,2-DCA degradation under the influence of salt water intrusion.

The aim of this study was to evaluate the impact of chloroethenes and 1,2-DCP on 1,2-DCA dechlorination using enrichment cultures containing Dehalococcoides, Geobacter, and Dehalogenimonas as the known OHRB. The prime focus was to obtain an improved understanding of 1,2-DCA dechlorination, which is the most abundant chlorinated organic contaminant worldwide (Field and Sierra-Alvarez 2004). 1,2DCA can be dihaloeliminated to ethene by diverse OHRB including members of the genera Dehalococcoides (MaymóGatell et al. 1999; Parthasarathy et al. 2015; Wang and He 2013), Geobacter (Duhamel and Edwards 2006), Dehalogenimonas (Maness et al. 2012), Desulfitobacterium (Low et al. 2019; Marzorati et al. 2007), and Dehalobacter (Grostern and Edwards 2009). 1,2-DCA has been found to coexist with chloroethenes and/or chloropropanes at many contaminated sites (Dillehay et al. 2014; Mayer-Blackwell et al. 2016). Despite some reports on suppression of 1,2-DCA dechlorination by co-occurring chloroethenes, chloroethanes, and bromoethanes (Dillehay et al. 2014; Mayer-Blackwell et al. 2016; Yu et al. 2013), comprehensive studies on the interaction between 1,2-DCA, 1,2-DCP, and chloroethenes with respect to their dechlorination in complex organohalide-respiring microbial consortia are limited.

In this study, in cultures amended with one or two chlorinated compounds (1,2-DCA with either tetrachloroethene (PCE), cDCE, VC, or 1,2-DCP), the impact of cocontaminants on OHRB was investigated by quantifying $16 \mathrm{~S}$ ribosomal RNA (rRNA) genes of known OHRB. The dechlorination reactions were approximated with MichaelisMenten kinetics taking into account competitive inhibition. Parameter estimation was performed using AMALGAM (Vrugt and Robinson 2007), a multi-objective, multi-method (ensemble) evolutionary optimization procedure to account for the high correlation among the parameters describing dechlorination kinetics and the existence of multiple solutions. Results showed that all applied chlorinated compounds inhibited 1,2-DCA dechlorination, whereas 1,2-DCA had no pronounced inhibitory effect on the dechlorination of other chlorinated compounds.

\section{Materials and methods}

\section{Chemicals}

1,2-DCA, chloroethenes, 1,2-DCP, ethene, and propene were purchased from Sigma-Aldrich, and were used directly in the 
following experiments. Lactate stock solution (1 M) was prepared from $60 \%$ sodium DL-lactate solution (Sigma-Aldrich). Other organic and inorganic chemicals were of analytical grade and were used without further purification.

\section{Sediment collection and enrichment set-up}

Surface sediment samples (down to $15 \mathrm{~cm}$ below surface) were collected from a wetland in Estarreja, Portugal. This site has a long history of contamination with agrochemical and fine chemistry effluents (Carvalho et al. 2005). Sediment enrichment cultures were set up in 120-mL serum bottles using $10 \mathrm{~g}$ of wet sediment and $50 \mathrm{~mL}$ of an anoxic medium as described previously (Stams et al. 1993). Resazurin $(0.005 \mathrm{~g} / \mathrm{L})$ and $\mathrm{Na}_{2} \mathrm{~S} \cdot 9 \mathrm{H}_{2} \mathrm{O}(0.48 \mathrm{~g} / \mathrm{L})$ were added as redox indicator and reducing reagent, respectively. The headspace of the bottles was exchanged with $\mathrm{N}_{2}$ and $\mathrm{CO}_{2}(80: 20 \%, 140 \mathrm{kPa})$. Lactate $(3 \mathrm{mM})$ was used as the carbon source and electron donor. The electron acceptors for the sediment cultures were PCE $(10 \mu \mathrm{mol} / \mathrm{bottle}$, designated EA culture) and PCE plus 1,2-DCA (10 $\mu \mathrm{mol} / \mathrm{bottle}$ each, designated EB culture). The bottles were sealed with Teflon lined butyl rubber stoppers and aluminum crimp caps (GRACE, MD, USA) and incubated statically in the dark at $20{ }^{\circ} \mathrm{C}$. The EA and EB sediment cultures were spiked 21 times with their respective chlorinated electron acceptors during enrichment. After dechlorination of each spike of the chlorinated substrates to ethene, the headspace of the cultures was flushed with $\mathrm{N}_{2}$ and $\mathrm{CO}_{2}$ (80:20\%) for three times ( $3 \mathrm{~min}$ for each run and $10 \mathrm{~min}$ rest in between each flushing cycle) before re-amendment of the respective chlorinated substrate(s). In the last three spikes, the concentration of each chlorinated substrate(s) was increased stepwise from $10 \mu \mathrm{mol} / \mathrm{bottle}$ to 25 and $40 \mu \mathrm{mol} / \mathrm{bottle}$. To avoid toxicity of the chlorinated compounds in the following experiments, each single chlorinated substrate was added at $25 \mu \mathrm{mol} / \mathrm{bottle}$ unless otherwise stated.

Sediment-free cultures were obtained by transferring the EA and EB sediment cultures as the following: a 5\% slurry from the EA and EB sediment cultures was transferred into bottles containing fresh anoxic medium with lactate (5 $\mathrm{mM}$ ) and single or double chlorinated substrates, i.e., 1,2DCA either alone or with PCE, cDCE, or VC (Fig. 1(a)). Each transfer culture was amended with three spikes of the respective chlorinated substrate(s), and then diluted to duplicate cultures (50\% inoculum, Fig. 1(b)) and amended with another three spikes of the respective chlorinated substrate(s). In parallel, a 5\% slurry from the EA and EB sediment cultures was also transferred into bottles containing 1,2-DCP (10 $\mu \mathrm{mol} / \mathrm{bottle})$. Only the EA transfer culture showed 1,2-DCP dechlorination. Therefore, only this culture was subsequently transferred (5\% inoculum) to fresh anoxic medium with either 1,2-DCP, 1,2-DCA, or a mixture of 1,2-DCP and 1,2-DCA (Fig. 1(c)). After depletion of three spikes of the respective chlorinated substrate, these cultures were diluted to duplicate cultures (50\% inoculum, Fig. 1(d)) and amended with another three spikes of the respective chlorinated substrate(s).

The dechlorination pattern of the last (third) spike of chlorinated substrates(s) (Fig. 1(b, d)) was used for kinetic modeling. To study the relief of inhibition by different chlorinated compounds on 1,2-DCA dechlorination, the cultures amended with double chlorinated substrates were subsequently spiked only with 1,2-DCA.

\section{DNA extraction and quantitative PCR}

After dechlorination of each spike of the chlorinated compounds during the last three spikes (amended respectively with 10,25 , and $40 \mu \mathrm{mol} /$ bottle each compound) in the EA and EB sediment cultures (Fig. 1), $2 \mathrm{~mL}$ slurry samples were taken for DNA extraction. After dechlorination of the third spike of chlorinated compounds in the sedimentfree transfer cultures that were used for kinetic modeling, 2 $\mathrm{mL}$ samples were also taken for DNA extraction. DNA was extracted using a DNeasy PowerSoil Kit (QIAGEN, Hilden, Germany) following the manufacturer's instructions. The copy number of $16 \mathrm{~S}$ rRNA genes was determined by real-time quantitative PCR (qPCR) with primers targeting total bacteria (Muyzer et al. 1993) and OHRB including Dehalococcoides (Smits et al. 2004), Geobacter (Amos et al. 2007), Dehalogenimonas (Chen et al. 2014), Dehalobacter (Smits et al. 2004), Desulfitobacterium (Smits et al. 2004), and Sulfurospirillum (Sutton et al. 2015) (Table S1). Assays were performed in triplicates using a CFX384 Real-Time system in a C1000 Thermal Cycler (Bio-Rad Laboratories, USA) with $\mathrm{iQ}^{\mathrm{TM}}$ SYBR Green Supermix (Bio-Rad Laboratories, USA).

\section{Analytical methods}

Chloroethenes, 1,2-DCA, 1,2-DCP, ethene, and propene were analyzed using a gas chromatograph-mass spectrometer (GCMS) composed of a Trace GC Ultra (Thermo Fisher Scientific, Waltham, MA, USA) equipped with an Rt@-Q-BOND column (Retek, PA, USA) and a DSQ MS (Thermo Fisher Scientific). Helium was used as a carrier gas with a flow rate of $2 \mathrm{ml} \mathrm{min}^{-1}$. The inlet temperature was $100{ }^{\circ} \mathrm{C}$. The split ratio was 30 . The temperature program of the column was 40 ${ }^{\circ} \mathrm{C}$ hold for $1 \mathrm{~min}$, followed by an increase at $40{ }^{\circ} \mathrm{C} \mathrm{min}{ }^{-1}$ to $260{ }^{\circ} \mathrm{C}$ and hold for $1.5 \mathrm{~min}$.

\section{Modeling and parameter estimation}

Reductive dechlorination of the chloroethenes in cultures shown in Fig. 1(b) and (d) was modeled using a 


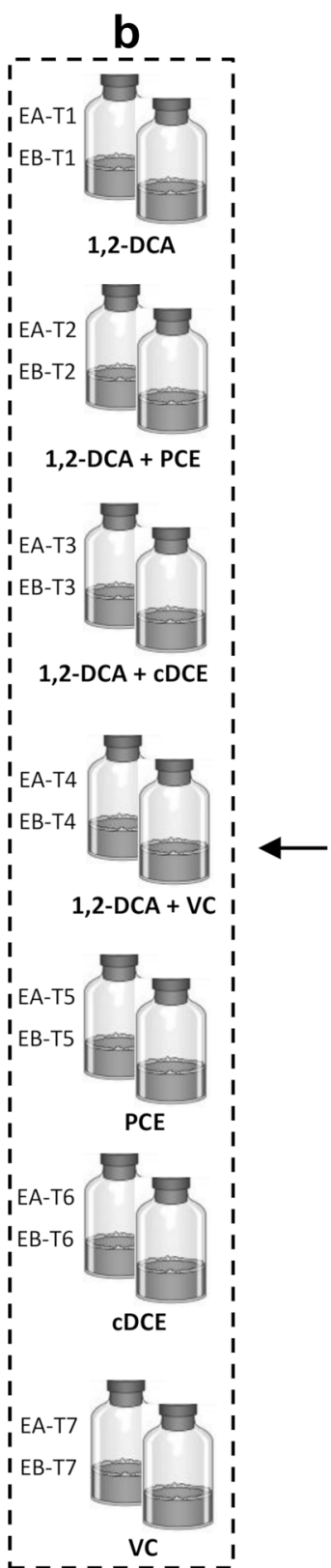

Fig. 1 Schematic representation of the experimental set-up. Cultures in box a received inoculum from either EA or EB culture. Cultures in dashed boxes $(\mathbf{b}, \mathbf{d})$ were used for the kinetic study. The EB culture

Michaelis-Menten model with competitive inhibition following Eq. (1)

$$
r_{n}=\frac{k_{\max , n} C_{n}}{C_{n}+K_{\mathrm{s}, n}\left(1+\sum_{i=1}^{x} \frac{C_{n+\mathrm{i}}}{I_{n+i}}\right)}
$$

where $r_{n}\left(\mu \mathrm{M} \mathrm{d}^{-1}\right)$ is the dechlorination rate that depends on the respective chlorinated compound, $k_{\max , n}$ $\left(\mu \mathrm{M} \mathrm{d}^{-1}\right)$ is the compound-specific maximum utilization rate or degradation constant, $K_{\mathrm{s}, n}(\mu \mathrm{M})$ is the compound-specific half-velocity constant, $I_{n}(\mu \mathrm{M})$ is the compound-specific was not able to dechlorinate 1,2-DCP and hence not used for the kinetic study performed using cultures in box $\mathbf{d}$

competitive inhibition rate, and $C_{n}(\mu \mathrm{M})$ is the aqueous concentration of the chlorinated compounds. The index $i$ represents the number of parent compounds considered in the dechlorination during competitive inhibition that varied with enrichment set-up.

Dihaloelimination of 1,2-DCA to ethene and of 1,2-DCP to propene were also modeled using Eq. (1). In the enrichment cultures containing both chloroethenes/1,2-DCP and 1,2DCA, competitive inhibition between 1,2-DCA and the other compounds was also included in the models.

As the Michaelis-Menten parameters describing dechlorination are highly correlated, model calibration was performed 
using AMALGAM (Vrugt and Robinson 2007), a multi-objective, multi-method (ensemble) evolutionary optimization procedure executable in MATLAB. AMALGAM attempts to find for each culture a set of optimal solutions, i.e., an ensemble of optimized kinetic parameters. These solutions have to adhere to the Pareto-principle, which means that all objectives (here, concentrations of the individual dechlorination products) must be met with equal efficiency. The number of Pareto-efficient solutions depends on model complexity and the size of the parameter space as well as the number of model runs and varies for each culture. As such we chose to provide in the results section ranges based on the 50 best parameter combinations of each culture. These were ranked according to their Euclidean distance to the zero-objective point of our $n$-dimensional space, where $n$ is the number of culture-specific objectives. Using a compromise solution (Werisch et al. 2014; Wöhling et al. 2008), the parameter set representing the solution with the smallest Euclidean distance on the Pareto front to the zeroobjective point was then utilized to create graphical representations as previously outlined (Schneidewind et al. 2014).

All cultures were modeled individually, and models were calibrated on the data obtained from the third spike, during which stable dechlorination patterns were noted. Initially, the cultures with the simplest dechlorination sequences (i.e., VC to ethene or 1,2-DCA to ethene) were modeled, and subsequently model complexity was gradually increased by including additional dechlorination reactions (i.e., cDCE to $\mathrm{VC}$ to ethene, etc.). Following this procedure, additional prior information could be used for more complex models to decrease the parameter space (defined by user-set upper and lower boundaries) from which AMALGAM retrieves optimal solutions. Initial boundary values were based on literature (Garant and Lynd 1998; Haest et al. 2010a; Haston and McCarty 1999; MayerBlackwell et al. 2016; Schaefer et al. 2009; Schneidewind et al. 2014; Yu and Semprini 2004) for chloroethenes and 1,2-DCA. For 1,2-DCP, boundary conditions were derived from simple Lineweaver-Burk plots (Lineweaver and Burk 1934). Dimensionless, species-dependent Henry coefficients at $20^{\circ} \mathrm{C}$ were used to account for volatilization of the chlorinated compounds in the cultures $(\mathrm{PCE}=0.711, \mathrm{TCE}=0.419, \mathrm{cDCE}=$ $0.182, \mathrm{VC}=1.075,1,2-\mathrm{DCA}=0.054,1,2-\mathrm{DCP}=0.123$, ethene $=7.108$, propene $=8.923$ ) (Mackay and Shiu 1981; Sander 2015; Staudinger and Roberts 2001).

\section{Results}

\section{Reductive dechlorination and dynamics of OHRB in the original sediment cultures}

1,2-DCA (10-40 $\mu \mathrm{mol} /$ bottle) was stoichiometrically converted to ethene in the EA and EB sediment cultures without production of any chlorinated intermediates indicating 1,2-DCA dihaloelimination (Fig. 2a). Besides 1,2-DCA, each spike of PCE $(10-40 \mu \mathrm{mol} /$ bottle) in the EB culture was concurrently dechlorinated to ethene (Fig. 2c). Dehalococcoides $\left(10^{7}-10^{8}\right.$ 16S rRNA gene copies/mL), Geobacter $\left(\sim 10^{8} 16 \mathrm{~S}\right.$ rRNA gene copies/mL), and Dehalogenimonas $\left(10^{6}-10^{7} 16 \mathrm{~S}\right.$ rRNA gene copies $/ \mathrm{mL}$ ) were the predominant OHRB in the EA culture (Fig. 2b). In contrast, Dehalococcoides $\left(10^{8}-10^{9} 16 \mathrm{~S}\right.$ rRNA gene copies $/ \mathrm{mL}$ ) was the predominant OHRB in the EB culture, and Geobacter and Dehalogenimonas numbers were $10^{7}$ and $10^{4} 16 \mathrm{~S}$ rRNA gene copies/mL, respectively (Fig. 2d). The $16 \mathrm{~S}$ rRNA gene numbers of Dehalobacter, Desulfitobacterium, and Sulfurospirillum in both EA and EB sediment cultures were below $10^{6}$ copies $/ \mathrm{mL}$, representing less than $0.1 \%$ of the total bacterial $16 \mathrm{~S}$ rRNA gene number (Fig. $2 b$ and $\mathrm{d}$ ). The $16 \mathrm{~S}$ rRNA gene copy numbers of OHRB were rather stable during the three spikes of chlorinated compounds (Fig. 2b and d). Hence, for the subsequent sediment-free transfer cultures, qPCR analysis was performed only at the end of the last (third) spike of the chlorinated compound(s).

\section{Dechlorination and co-contaminant effect of 1,2-DCA and chloroethenes}

1,2-DCA dechlorination was maintained in the EA and EB sediment-free transfer cultures (cultures EA-T1 and EB-T1, Figs. 3a and 4a). Moreover, PCE, cDCE, and VC were completely dechlorinated to ethene in both EA and EB transfer cultures (cultures EA-T5-T7, Fig. 3e-g, and cultures EBT5-T7, Fig. 4e-g), although PCE was not amended in the original EA culture. In EA and EB transfer cultures amended with same amounts ( $25 \mu \mathrm{mol} / \mathrm{bottle}$ each spike) of 1,2-DCA and either PCE, cDCE, or VC, dechlorination of 1,2-DCA was inhibited due to decreased dechlorination rate by the chloroethenes (cultures EA-T2-T4, Fig. 3b-d and cultures EB-T2-T4, Fig. 4b-d), especially when cDCE was present as co-contaminant, where 1,2-DCA dechlorination did not start until cDCE was depleted (culture EA-T3 Fig. 3c and culture EB-T3, Fig. 4c). During dechlorination of the third spike, the time to complete dechlorination of 1,2-DCA increased from around 3 days (Fig. S3-S4) to 7-14 days (Fig. S5-S10) in EA transfer cultures in the presence of chloroethenes, and from around 3 days (Fig. S23-S24) to 68 days (Fig. S25-S30) in EB transfer cultures. After the third spike, when the cultures with co-contaminants (Figs. 3b-d and 4b-d) were amended with only 1,2-DCA, its dechlorination was completed in 2-5 days in both EA and EB transfer cultures (Fig. S2A-F), whereas the same amount of 1,2-DCA was dechlorinated in 6-14 days in the presence of chloroethenes. In contrast, no pronounced inhibitory effect of 1,2-DCA on chloroethene dechlorination was observed. Dechlorination of chloroethenes was comparable between cultures where chloroethenes were amended as a single compound (cultures EA-T5-T7, Fig. 3e-g, S11-S16, and cultures EB-T5-T7, Fig. 
Fig. 2 Reductive dechlorination of 1,2-DCA and PCE in the sediment cultures EA (a) and EB (c) during the last three spikes, and 16S rRNA gene copy numbers of total bacteria, Dehalococcoides, Geobacter, Dehalogenimonas, Dehalobacter, Desulfitobacterium, and Sulfurospirillum at the end of each spike (b, d). The arrows in panels $\mathbf{a}$ and $\mathbf{c}$ indicate re-spike of the chlorinated substrates. Error bars of the qPCR values indicate standard deviations of triplicate qPCRs performed on one sample of each culture
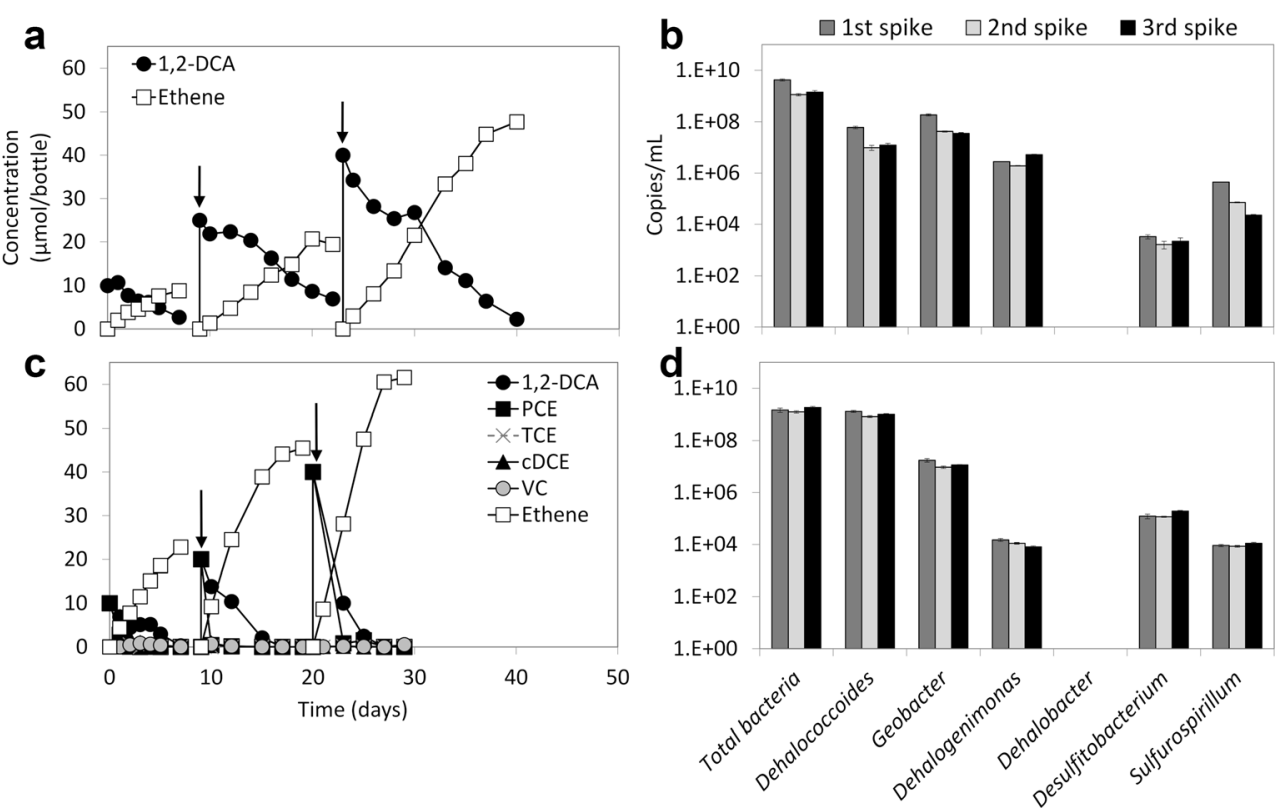

4e-g, S31-S36) and cultures where they were added together with 1,2-DCA (cultures EA-T2-T4, Fig. 3b-d, S5-S10 and cultures EB-T2-T4, Fig. 4b-d, S25-S30).

The 16S rRNA gene copy number of Dehalococcoides in EA transfer cultures $\left(\sim 10^{8}\right.$ copies $/ \mathrm{mL}$, Fig. $\left.3 \mathrm{~h}-\mathrm{j}\right)$ was about one order of magnitude higher than in the original EA sediment culture (Fig. 2b), whereas the 16S rRNA gene copy numbers of Dehalococcoides in the EB culture $\left(10^{8}-10^{9}\right.$ copies/mL, Fig. 2d) and its transfer cultures (Fig. 4h-j) were similar. In EA transfer cultures, Dehalogenimonas 16S rRNA gene copy numbers were 1-3 orders of magnitude higher in the cultures fed 1,2-DCA, VC, or 1,2-DCA plus chloroethenes (PCE, cDCE, VC) than in the cultures fed only PCE or cDCE (Fig. $3 \mathrm{~h}-\mathrm{j}$ ). The $16 \mathrm{~S}$ rRNA gene copy numbers of Dehalogenimonas were below $10^{3}$ copies $/ \mathrm{mL}$ in the EB transfer cultures (Fig. $4 \mathrm{~h}-\mathrm{j}$ ), in line with the pattern in the EB sediment culture (Fig. 2d). The 16S rRNA gene copy numbers of Geobacter in the EA and EB transfer cultures were $10^{7}-10^{8}$ copies $/ \mathrm{mL}$ (Figs. $3 \mathrm{~h}-\mathrm{j}$ and $4 \mathrm{~h}-\mathrm{j}$ ).

\section{Dechlorination and co-contaminant effect of 1,2-DCA and 1,2-DCP}

The original EA and EB sediment cultures had not been amended with 1,2-DCP. To study the co-contaminant effect between 1,2-DCA and 1,2-DCP, EA and EB cultures were first transferred (5\% inoculum) to fresh media containing only 1,2DCP $(10 \mu \mathrm{mol} /$ bottle $)$. During 70 days of incubation, more than $90 \%$ of the 1,2-DCP in the EA transfer culture was dechlorinated to propene (Fig. S1), whereas no 1,2-DCP dechlorination was observed in the EB transfer culture (data not shown). Therefore, the EA transfer culture fed 1,2-DCP was used to study the co-contaminant effect (Fig. 1d). In the subsequent transfer cultures, 1,2-DCP dechlorination was stably maintained (cultures EA-T10, Fig. 5c), while 1,2-DCA was also dechlorinated (cultures EA-T8, Fig. 5a). Similar to the cocontaminant effect between 1,2-DCA and chloroethenes, 1,2DCA dechlorination was inhibited in the transfer cultures concurrently amended with 1,2-DCP, whereas no obvious inhibitory effect of 1,2-DCA on 1,2-DCP dechlorination was observed (culture EA-T9, Fig. 5b). Specifically, the time to complete dechlorination of 1,2-DCA was increased from around 3 days (Fig. S17-S18) to 9 days in the presence of 1,2-DCP (Fig. S19-S20), while 1,2-DCP dechlorination was not inhibited (Fig. S19-S22). When these cultures were amended only with 1,2-DCA, its dechlorination was completed in 2 days (Fig. S2G), which was strongly enhanced compared with its dechlorination in the presence of 1,2-DCP (same amount of 1,2-DCA was dechlorinated in 9 days) (Fig. 5b). Dehalococcoides $\left(\sim 10^{8}\right.$ 16S rRNA gene copies $/ \mathrm{mL})$, Geobacter $\left(\sim 10^{7} 16 \mathrm{~S}\right.$ rRNA gene copies $/ \mathrm{mL})$, and Dehalogenimonas $\left(10^{6}-10^{7} 16 \mathrm{~S}\right.$ rRNA gene copies $/ \mathrm{mL}$ ) were the predominant known OHRB in these transfer cultures, similar to EA transfer cultures amended with 1,2DCA and chloroethenes (Figs. 3h-g and 5d).

\section{Dechlorination kinetics}

Table 1 provides a summary of parameter ranges for all compounds as compared with the values from previous studies. Those parameter ranges were obtained by modeling dechlorination after the third spike only. Maximum and minimum values of the 50 best parameter combinations for individual cultures are shown in Table S2. $K_{s}$ and $I$ values of the chloroethenes are in the same range as in the previous studies listed in Table 1. A comparison of values of $k_{\max }$ is less straight forward as many studies provide $k_{\max }$ in units related to the bacterial cell or protein 


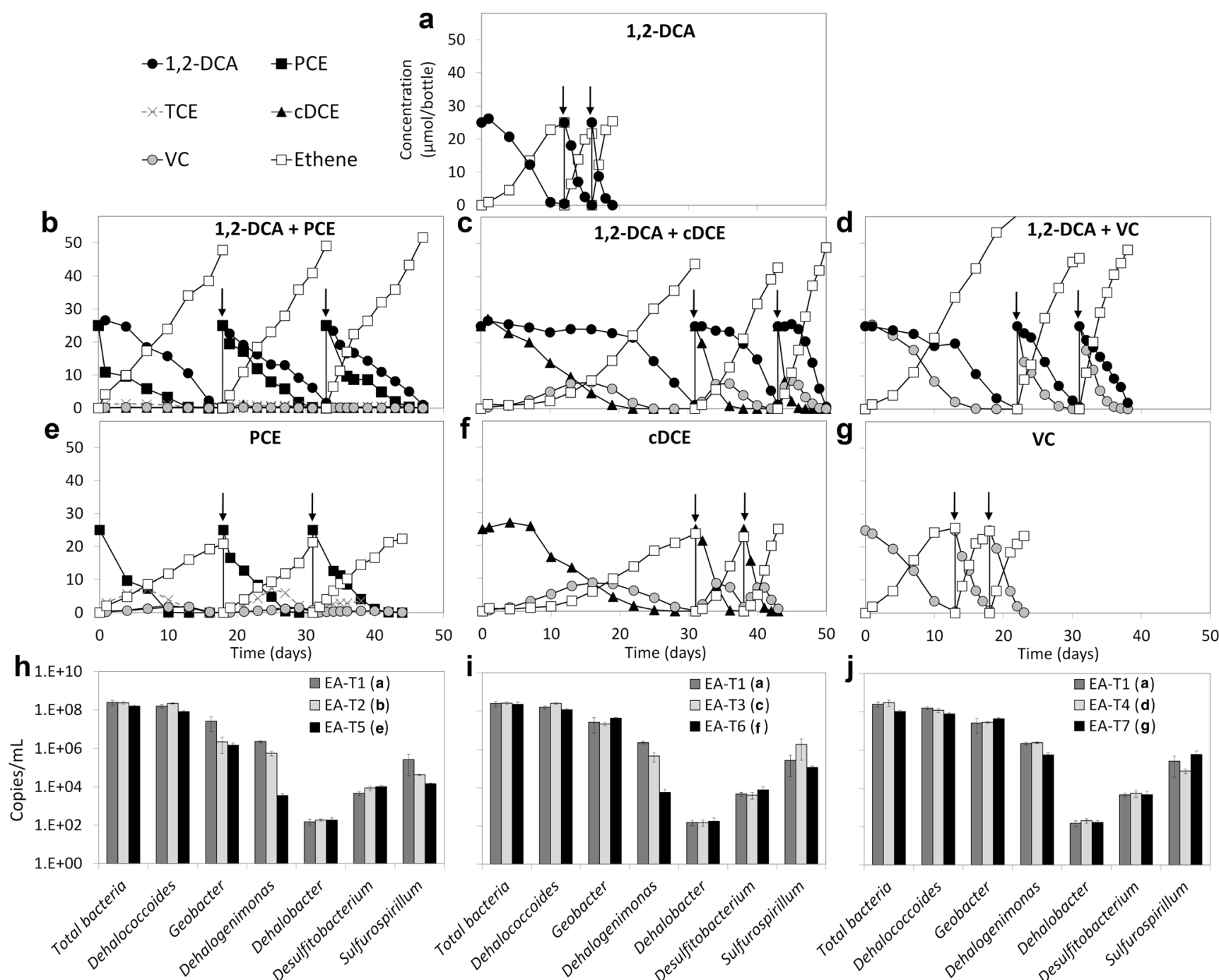

Fig. 3 Reductive dechlorination of 1,2-DCA (EA-T1, a), 1,2-DCA plus PCE (EA-T2, b), 1,2-DCA plus cDCE (EA-T3, c), 1,2-DCA plus VC (EA-T4, d), PCE (EA-T5, e), cDCE (EA-T6, f), and VC (EA-T7, g) in the sediment-free enrichment cultures obtained from EA sediment culture, and 16S rRNA gene copy numbers of total bacteria, Dehalococcoides, Geobacter, Dehalogenimonas, Dehalobacter, Desulfitobacterium, and

mass. $k_{\max }$ estimates for PCE and TCE obtained here are similar to those in Haston and McCarty (1999) and Schneidewind et al. (2014), while $k_{\max }$ estimates for CDCE and VC in this study were about one order of magnitude higher.

A comparison of parameter estimates for 1,2-DCP dechlorination was not possible due to the lack of kinetic models in literature. Parameter estimates of 1,2-DCA were comparable with those found by Mayer-Blackwell et al. (2016). However, in our study, they span a rather wide range, defined by cultures EA-T9, where concurrent dechlorination of 1,2-DCA and 1,2DCP occurred (see Fig. S19 and S20 and Table S2), as further discussed below.

In general, modeled and observed results showed a good fit for cultures amended with a single chlorinated compound (e.g., EA-T1, EA-T7, Figs. S3-S4, S15-S16) indicated by low root-
Sulfurospirillum at the end of the third spike in these cultures $(\mathbf{h}, \mathbf{i}, \mathbf{j})$. Each concentration value represents the average measured from duplicate cultures. The arrows in panels $\mathbf{a}-\mathbf{g}$ indicate re-spike of the chlorinated substrates. Error bars were not included in panels $\mathbf{a}-\mathbf{g}$ for clarity. Error bars of the qPCR values indicate standard deviations of triplicate qPCRs performed on one sample of each of the duplicate cultures $(n=2 \times 3)$

mean-square errors (RMSE, data not shown). Model fits decreased (higher RMSE) for the cultures with more complex reaction networks (e.g., EB-T2, Fig. S25-S26). Duplicate batches showed comparable parameter ranges, and parameters differed by less than one order of magnitude. A notable exception is experiment EA-T9 (Fig. S19, S20) where one of the replicate cultures (culture A) showed relatively narrow parameter ranges for 1,2-DCA, whereas parameter ranges for culture $\mathrm{B}$ for 1,2-DCA varied by several orders of magnitude.

\section{Discussion}

The present study revealed inhibition of 1,2-DCA dechlorination in the presence of chloroethenes and 1,2-DCP using 


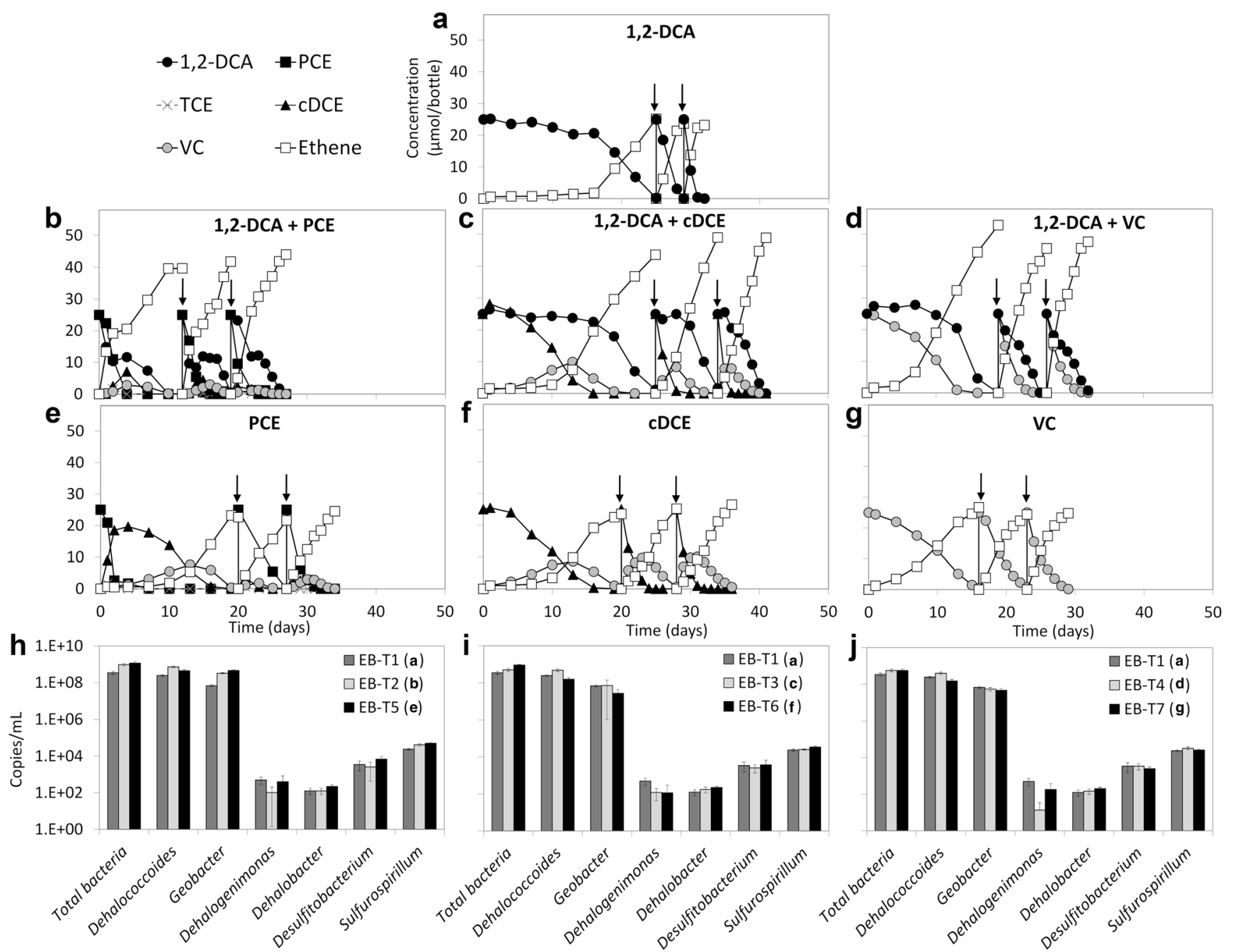

Fig. 4 Reductive dechlorination of 1,2-DCA (EB-T1, a), 1,2-DCA plus PCE (EB-T2, b), 1,2-DCA plus cDCE (EB-T3, c), 1,2-DCA plus VC (EB-T4, d), PCE (EB-T5, e), cDCE (EB-T6, f), and VC (EB-T7, g) in the sediment-free enrichment cultures obtained from EB sediment culture, and 16S rRNA gene copy numbers of total bacteria, Dehalococcoides, Geobacter, Dehalogenimonas, Dehalobacter, Desulfitobacterium, and Sulfurospirillum at the end of the third spike in

organohalide-respiring microbial consortia obtained from a wetland contaminated with agrochemical products. Among the tested chlorinated substrates, $\mathrm{CDCE}$ showed the strongest inhibitory effect on 1,2-DCA dechlorination. Dechlorination of 1,2-DCA started only when cDCE was completely depleted (culture EA-T3, Fig. 3c and culture EB-T3, Fig. 4c). This is consistent with previous findings showing that cDCE strongly inhibited 1,2-DCA dechlorination using a continuous enrichment culture containing Dehalococcoides (Mayer-Blackwell et al. 2016). We also noted inhibition of 1,2-DCA dechlorination in the presence of PCE, VC, and 1,2-DCP, further supported by the decreased $k_{\max , \mathrm{DCA}}$ and increased $K_{\mathrm{s}, \mathrm{DCA}}$ values compared with the cultures amended with 1,2-DCA only. Notably, the inhibitory pattern of PCE, VC, and 1,2-DCP on 1,2-DCA dechlorination was different from that of cDCE. In cultures these cultures $(\mathbf{h}, \mathbf{i}, \mathbf{j})$. Each concentration value represents the average measured from duplicate cultures. The arrows in panels a-g indicate respike of the chlorinated substrates. Error bars were not included in panels $\mathbf{a}-\mathbf{g}$ for clarity. Error bars of the qPCR values indicate standard deviations of triplicate qPCRs performed on one sample of each of the duplicate cultures $(n=2 \times 3)$

containing 1,2-DCA with either PCE, VC, or 1,2-DCP as cocontaminants, the cultures concurrently dechlorinated both amended chlorinated compounds. However, delayed dechlorination of 1,2-DCA due to a decreased dechlorination rate was observed during concurrent dechlorination of 1,2-DCA and PCE (culture EA-T2, Fig. 3b and culture EB-T2, Fig. 4b), probably because of the $\mathrm{CDCE}$ production from $\mathrm{PCE}$.

The observed inhibitory effect of VC on 1,2-DCA dechlorination is in contrast to what was reported by MayerBlackwell et al. (2016) using a continuous enrichment culture containing Dehalococcoides where VC had a negligible inhibitory effect on 1,2-DCA dechlorination. Interestingly, Mayer-Blackwell et al. (2016) found that long-term exposure of the continuous culture to 1,2-DCA shifted the Dehalococcoides population, leading to 

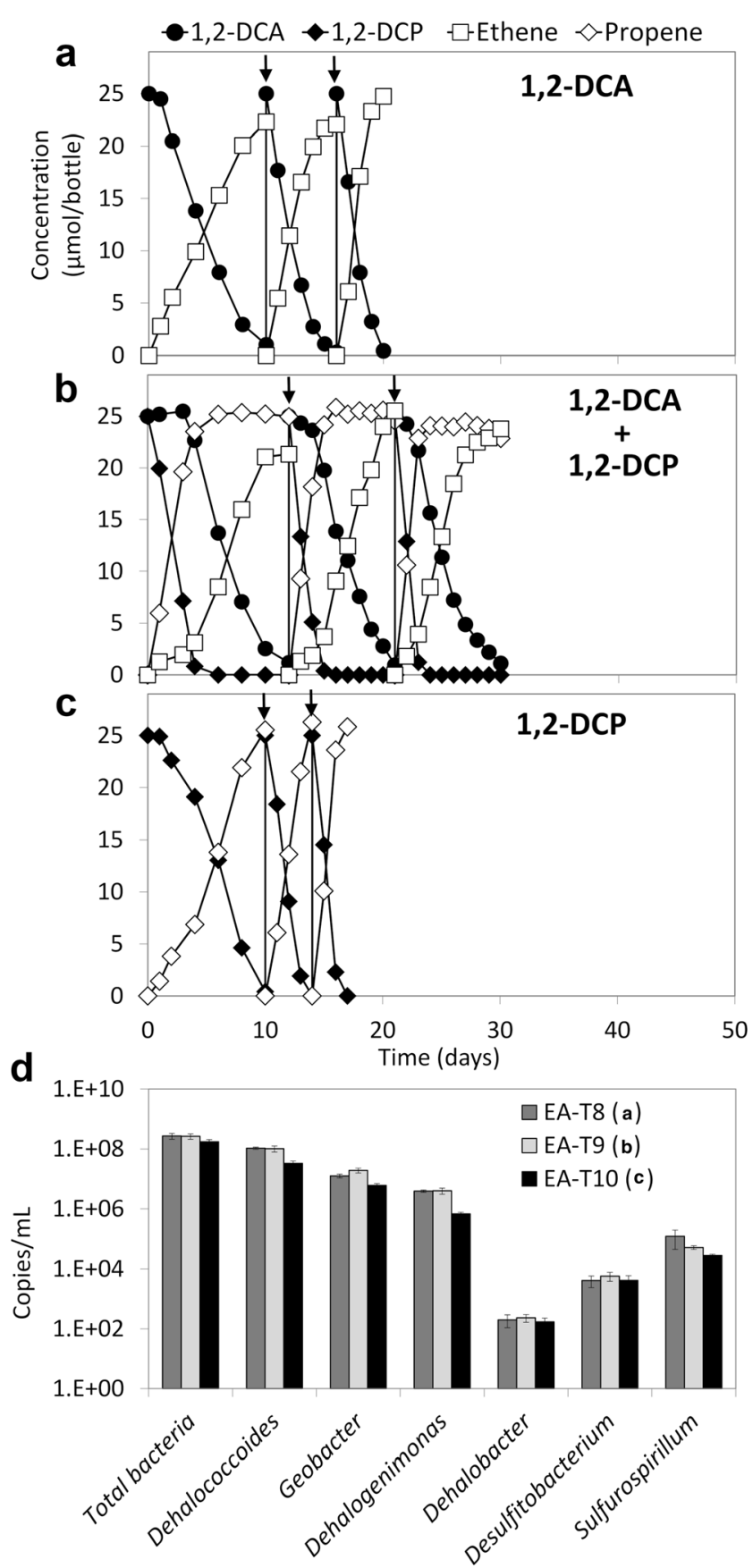

Fig. 5 Reductive dechlorination of 1,2-DCA (EA-T8, a), 1,2-DCA plus 1,2-DCP (EA-T9, b), 1,2-DCP (EA-T10, c) in sediment-free cultures derived from the EA transfer culture amended with 1,2-DCP, and $16 \mathrm{~S}$ rRNA gene copy numbers of total bacteria, Dehalococcoides, Geobacter, Dehalogenimonas, Dehalobacter, Desulfitobacterium, and Sulfurospirillum at the end of the third spike in these cultures (d). Each concentration value represents the average measured from duplicate cultures. The arrows in panels a-c indicate re-spike of the chlorinated substrates. Error bars were not included in panels a-c for clarity. Error bars of the qPCR values indicate standard deviations of triplicate qPCRs performed on one sample of each of the duplicate cultures $(n=2 \times 3)$

diminished VC dechlorinating ability. In contrast, we found no inhibitory effect of 1,2-DCA on the dechlorination of chloroethenes and 1,2-DCP in batch cultures.

Based on the data presented here, it is likely that the original sediments in EA and EB cultures contained at least two different Dehalococcoides populations. The 1,2-DCP dechlorinating population was maintained during enrichment in the presence of 1,2DCA (EA culture) but likely lost during incubation in the presence of 1,2-DCA plus PCE (EB culture). This suggests that 1,2DCA but not PCE was also a growth substrate for the 1,2-DCP dechlorinating Dehalococcoides population, whereas PCE was likely a substrate for the other Dehalococcoides population. The EA transfer cultures were also able to dechlorinate PCE (Fig. $3 \mathrm{~b}, \mathrm{e})$. This indicates that feeding 1,2-DCA alone in the EA sediment culture also maintained the Dehalococcoides population capable of PCE dechlorination, and therefore, 1,2-DCA was also a growth substrate for the PCE-dechlorinating Dehalococcoides population. Feeding 1,2-DCA plus PCE likely promoted selective growth of the 1,2-DCA/PCE-dechlorinating Dehalococcoides over the 1,2-DCA/1,2-DCP dechlorinating Dehalococcoides. This is also likely the reason for the loss of Dehalogenimonas in the EB culture, as Dehalogenimonas is known to dechlorinate 1,2-DCA and 1,2-DCP via dihaloelimination but does not dechlorinate PCE (Bowman et al. 2013; Moe et al. 2009). Accordingly, Dehalogenimonas was only maintained in the EA transfer cultures amended with substrates known to support Dehalogenimonas growth (e.g., 1,2DCA or 1,2-DCA plus VC or 1,2-DCA plus 1,2-DCP) (Maness et al. 2012; Martín-González et al. 2015; Moe et al. 2016; Yang et al. 2017) (Fig. 3h-j). Notably, feeding solely PCE or cDCE that are not known growth substrates for Dehalogenimonas decreased Dehalogenimonas 16S rRNA gene copy numbers by $1-$ 3 orders of magnitude (Fig. 3h, i), whereas feeding solely VC or 1,2-DCP that are known substrates to support Dehalogenimonas growth (Bowman et al. 2013; Moe et al. 2009; Yang et al. 2017) did not strongly reduce its 16S rRNA gene copy numbers (Figs. $3 \mathrm{j}$ and $4 \mathrm{~d})$. Geobacter, which is known to dechlorinate PCE/ TCE (Sung et al. 2006), and 1,2-DCA (Duhamel and Edwards 2007), was stably maintained in both EA (Fig. 3h-j) and EB (Fig. $4 \mathrm{~h}-\mathrm{j}$ ) sediment-free transfer cultures. Our results indicate that the type of chlorinated substrate drives the selection of OHRB. Likewise, a recent study showed that a Dehalococcoides population shift was driven by different chlorinated electron acceptors in enrichment cultures containing Dehalococcoides and at a contaminated site (Pérez-de-Mora et al. 2018).

Michaelis-Menten kinetics was successfully used to model dechlorination after the third spike when dechlorination followed a concave pattern. Parameter estimates obtained here compare well with those obtained in previous studies (see Table 1). Different kinetic models could be more suited to model dechlorination after the first and the second spikes. Especially dechlorination after the first spike might be modeled more successfully using Monod kinetics (see also Schneidewind et al., 2014) to better account for an apparent lag phase before the onset of dechlorination. However, Monod 
Table 1 Range of parameter estimates obtained from modeling using a Michaelis-Menten kinetics approach in comparison with previous studies

\begin{tabular}{|c|c|c|c|c|c|c|c|}
\hline Parameter & Unit & This work & $\begin{array}{l}\text { Garant and } \\
\text { Lynd }(1998)^{\mathrm{a}}\end{array}$ & $\begin{array}{l}\text { Haston and } \\
\text { McCarty }(1999)^{\mathrm{b}}\end{array}$ & $\begin{array}{l}\text { Yu and Semprini } \\
(2004)^{\mathrm{c}}\end{array}$ & $\begin{array}{l}\text { Schneidewind } \\
\text { et al. }(2014)^{\mathrm{d}}\end{array}$ & $\begin{array}{l}\text { Mayer- } \\
\text { Blackwell } \\
\text { et al. }(2016)^{\text {e }}\end{array}$ \\
\hline$k_{\max , \mathrm{PCE}}$ & {$\left[\mu \mathrm{M} \mathrm{d}^{-1}\right]$} & $38.7-443.4$ & $15550^{\mathrm{f}}$ & 77 & $12.4 / 13.3^{\mathrm{g}}$ & & \\
\hline$K_{\mathrm{s}, \mathrm{PCE}}$ & {$[\mu \mathrm{M}]$} & $\leq 1.0$ & 70.7 & 0.1 & $1.6 / 3.9$ & & \\
\hline$I_{\mathrm{PCE}}$ & {$[\mu \mathrm{M}]$} & $3.7-370$ & 70.7 & & $1.6 / 3.9$ & & \\
\hline$k_{\max , \mathrm{TCE}}$ & {$\left[\mu \mathrm{M} \mathrm{d}^{-1}\right]$} & $39.5-1000$ & $9380^{\mathrm{f}}$ & 59 & $124 / 125^{\mathrm{g}}$ & $2.6-12$ & \\
\hline$K_{\mathrm{s}, \mathrm{TCE}}$ & {$[\mu \mathrm{M}]$} & $0.1-14$ & 17.4 & 1.4 & $1.8 / 2.8$ & $2.1-42$ & \\
\hline$I_{\mathrm{TCE}}$ & {$[\mu \mathrm{M}]$} & $3.7-370$ & 17.4 & & $1.8 / 2.8$ & $3.7-370$ & \\
\hline$k_{\max , \mathrm{cDCE}}$ & {$\left[\mu \mathrm{M} \mathrm{d}^{-1}\right]$} & $139.7-245.2$ & $5880^{\mathrm{f}}$ & 14 & $13.8 / 22^{\mathrm{g}}$ & $0.9-94.4$ & 2688 \\
\hline$K_{\mathrm{s}, \mathrm{cDCE}}$ & {$[\mu \mathrm{M}]$} & $<0.1-50.8$ & 11.9 & 3.3 & $1.8 / 1.9$ & $3.8-37.8$ & 8.5 \\
\hline$I_{\mathrm{cDCE}}$ & {$[\mu \mathrm{M}]$} & $3.7-370$ & 11.9 & & $1.8 / 1.9$ & $3.7-370$ & 9 \\
\hline$k_{\max , \mathrm{VC}}$ & {$\left[\mu \mathrm{M} \mathrm{d}^{-1}\right]$} & $127.4-161.0$ & $6670^{\mathrm{f}}$ & 13 & $2.4 / 8.1^{\mathrm{g}}$ & $0.4-14.4$ & \\
\hline$K_{\mathrm{s}, \mathrm{VC}}$ & {$[\mu \mathrm{M}]$} & $17.7-26.3$ & 383 & 2.6 & $62.6 / 60.2$ & $3.8-37.8$ & \\
\hline$I_{\mathrm{VC}}$ & {$[\mu \mathrm{M}]$} & $3.7-370$ & & & $62.6 / 60.2$ & & \\
\hline$k_{\max , \mathrm{DCA}}$ & {$\left[\mu \mathrm{M} \mathrm{d}^{-1}\right]$} & $<0.1-7535.8^{\mathrm{h}}$ & & & & & 960 \\
\hline$K_{\mathrm{s}, \mathrm{DCA}}$ & {$[\mu \mathrm{M}]$} & $<0.1-1032.5^{\mathrm{i}}$ & & & & & 127 \\
\hline$I_{\mathrm{DCA}}$ & {$[\mu \mathrm{M}]$} & $<0.1-370^{\mathrm{j}}$ & & & & & 45 \\
\hline$k_{\max , \mathrm{DCP}}$ & {$\left[\mu \mathrm{M} \mathrm{d}^{-1}\right]$} & $220.5-243.1$ & & & & & \\
\hline$K_{\mathrm{s}, \mathrm{DCP}}$ & {$[\mu \mathrm{M}]$} & $\leq 1.1$ & & & & & \\
\hline$I_{\mathrm{DCP}}$ & {$[\mu \mathrm{M}]$} & $1.2-370$ & & & & & \\
\hline
\end{tabular}

${ }^{\mathrm{a}}$ From Table 2 of the reference (competitive case)

${ }^{\mathrm{b}}$ From Table 2 of the reference

${ }^{\mathrm{c}}$ From Table 2 of the reference, first number EV, second number PM

${ }^{\mathrm{d}}$ From Table 2 of the reference

${ }^{\mathrm{e}}$ From Table 3 of the reference

${ }^{\mathrm{f}} \mu \mathrm{mol} / \mathrm{d}$ and $\mathrm{g}$ cells

${ }^{\mathrm{g}} \mu \mathrm{mol} / \mathrm{d}$ and mg protein

${ }^{\mathrm{h}, \mathrm{i}, \mathrm{j}}$ For 1,2 -DCA in batches without 1,2-DCP: $157.9 \leq k_{\max , \mathrm{DCA}} \leq 1428.9,2.9 \leq K_{\mathrm{s}, \mathrm{DCA}} \leq 1032.5,2.5 \leq I_{\mathrm{DCA}} \leq 250.0$

modeling was not used in this study due to limited information on microbial interactions (growth and decay patterns).

An interpretation of competitive inhibition is not straightforward from the obtained inhibition constants $I$ (Table S2). For example, cDCE seems to have a pronounced effect on 1,2DCA dechlorination only at starting concentrations of cDCE $\left(\sim I_{C D C E}\right)$. As soon as cDCE concentrations drop by a factor $\geq$ 10 , inhibition of 1,2-DCA dechlorination by cDCE becomes much less important. The calibrated inhibition constants suggest that $\mathrm{VC}$ is a stronger inhibitor on 1,2-DCA dechlorination than cDCE in all cultures except EB-T2 $\left(I_{V C}\right.$ is substantially below $\left.I_{C D C E}\right)$. However, $I_{V C}$ is largest in cultures EA-T4 and EB-T4 where no higher chlorinated compounds were present. A higher competitive inhibition constant indicates a smaller inhibitive effect. The parameter for VC probably lumps inhibition effects from higher chlorinated parent products when present, resulting in a higher simulated inhibition effect of VC in mixed compound tests.

In addition, interpreting parameter estimates (especially inhibition constants) obtained from cultures with multiple chlorinated compounds proved challenging, as strong crosscorrelation exists among the parameters of the MichaelisMenten kinetics. For example, we observed comparable $K_{\mathrm{s}}$ and $k_{\max }$ values for chloroethenes and 1,2-DCP in cultures with and without 1,2-DCA. In contrast, $k_{\max , \mathrm{DCA}}$ values decreased and $K_{\mathrm{s}, \mathrm{DCA}}$ values increased in multi-compound cultures compared with the cultures amended with 1,2-DCA only. This hints towards an effect of the chloroethenes and 1,2-DCP on 1,2DCA dechlorination. However, the best parameter combination did not put this effect in the inhibition constants, but rather in the dechlorination constants of 1,2-DCA itself. Another example is culture EA-T9: a small $K_{\mathrm{S}}$ of 1,2-DCA is counteracted by a small inhibition constant of 1,2-DCP in the duplicate cultures. In other words, the obtained parameters suggest that dechlorination of 1,2-DCA could occur at a maximal rate at low substrate concentration, but would then be more inhibited by 1,2DCP; or in contrast, the maximal dechlorination rate of 1,2DCA is not attained under the current experimental conditions but its dechlorination would be less inhibited by 1,2-DCP. However, both parameter combinations would yield a proper 
fit of the experimental observations, proving the nonuniqueness of the solution (Beven 2001).

The use of results from less complex culture set-ups in the refinement of the parameter space, from which AMALGAM choses viable solutions for more complex set-ups (e.g., EAT1, EA-T4 and EA-T6 for EA-T2), allowed us to reduce the uncertainty on the parameter estimates. However, the problem of non-uniqueness still remains, e.g., the existence of multiple parameter combinations producing an equally good fit. Uncertainty on the parameter estimates arises due to incomplete or insufficient information on the dechlorination processes in the individual cultures (e.g., information on microbial interactions or on necessary micro-nutrients).

In conclusion, the inhibitory effect of chloroethenes and 1,2-DCP on 1,2-DCA dechlorination identified in this study has important implications for understanding the persistence of 1,2-DCA at many contaminated sites. For effective bioremediation of such contaminated sites, it will be necessary to first remove potential inhibitors such as CDCE as well as its parent compounds PCE and TCE, which can delay 1,2-DCA dechlorination and even cause loss of 1,2-DCA/1,2-DCP dechlorinating Dehalococcoides and Dehalogenimonas populations. Further studies are needed to better understand the inhibitory mechanisms. Possible experimental approaches include identification of the genes and enzymes involved in 1,2DCA dichlorination, study the transcriptional regulation of these genes, and assess potential competitive inhibition of the enzymes. Theoretical modeling experiments that more rigorously look into the effect of the size of the parameter space or the use of different estimation algorithms could further improve our understanding of parameter/model uncertainty.

Acknowledgments The assistance of Rita Lopes and Olga Nunes (FEUP) and Nora B. Sutton (Wageningen University \& Research) is greatly appreciated.

Funding information This study was supported by the BE-BASIC funds (grant F08.004.01) from the Dutch Ministry of Economic Affairs, and the Gravitation grant (project 024.002.002) of the Netherlands Ministry of Education, Culture and Science, and the Netherlands Science Foundation (NWO). PP was supported by the China Scholarship Council (CSC).

\section{Compliance with ethical standards}

Conflict of interest The authors declare that they have no conflict of interest.

Ethical statement This article does not contain any studies with human participants performed by any of the authors.

Open Access This article is distributed under the terms of the Creative Commons Attribution 4.0 International License (http:// creativecommons.org/licenses/by/4.0/), which permits unrestricted use, distribution, and reproduction in any medium, provided you give appropriate credit to the original author(s) and the source, provide a link to the Creative Commons license, and indicate if changes were made.

\section{References}

Amos BK, Sung Y, Fletcher KE, Gentry TJ, Wu W-M, Criddle CS, Zhou J, Löffler FE (2007) Detection and quantification of Geobacter lovleyi strain SZ: implications for bioremediation at tetrachloroethene-and uranium-impacted sites. Appl Environ Microbiol 73(21):6898-6904. https://doi.org/10.1128/Aem.0121807

Atashgahi S, Lu Y, Smidt H (2016) Overview of known organohaliderespiring bacteria - phylogenetic diversity and environmental distribution. In: Adrian L, Löffler FE (eds) Organohalide-respiring bacteria. Springer, Berlin, pp 63-105

Atashgahi S, Lu Y, Zheng Y, Saccenti E, Suarez-Diez M, Ramiro-Garcia J, Eisenmann H, Elsner M, Stams AJM, Springael D, Dejonghe W, Smidt H (2017) Geochemical and microbial community determinants of reductive dechlorination at a site biostimulated with glycerol. Environ Microbiol 19(3):968-981. https://doi.org/10.1111/ 1462-2920.13531

Atashgahi S, Sanchez-Andrea I, Heipieper HJ, van der Meer JR, Stams AJM, Smidt H (2018) Prospects for harnessing biocide resistance for bioremediation and detoxification. Science 360(6390):743-746. https://doi.org/10.1126/science.aar3778

Beven K (2001) How far can we go in distributed hydrological modelling? Hydrol Earth Syst Sci 5(1):1-12

Bowman KS, Nobre MF, da Costa MS, Rainey FA, Moe WM (2013) Dehalogenimonas alkenigignens sp. nov., a chlorinated-alkanedehalogenating bacterium isolated from groundwater. Int J Syst Evol Microbiol 63(4):1492-1498. https://doi.org/10.1099/ijs.0. 045054-0

Brovelli A, Barry DA, Robinson C, Gerhard JI (2012) Analysis of acidity production during enhanced reductive dechlorination using a simplified reactive transport model. Adv Water Resour 43:14-27. https://doi.org/10.1016/j.advwatres.2012.04.001

Carvalho M, Ferreira Jorge R, Pacheco C, De Marco P, Castro P (2005) Isolation and properties of a pure bacterial strain capable of fluorobenzene degradation as sole carbon and energy source. Environ Microbiol 7(2):294-298. https://doi.org/10.1111/j.14622920.2004.00714.x

Chambon JC, Bjerg PL, Scheutz C, Bælum J, Jakobsen R, Binning PJ (2013) Review of reactive kinetic models describing reductive dechlorination of chlorinated ethenes in soil and groundwater. Biotechnol Bioeng 110(1):1-23. https://doi.org/10.1002/bit.24714

Chan WW, Grostern A, Löffler FE, Edwards EA (2011) Quantifying the effects of 1,1,1-trichloroethane and 1,1-dichloroethane on chlorinated ethene reductive dehalogenases. Environ Sci Technol 45(22): 9693-9702. https://doi.org/10.1021/es201260n

Chen J, Bowman KS, Rainey FA, Moe WM (2014) Reassessment of PCR primers targeting 16S rRNA genes of the organohalide-respiring genus Dehalogenimonas. Biodegradation 25(5):747-756. https:// doi.org/10.1007/s10532-014-9696-Z

Christ JA, Abriola LM (2007) Modeling metabolic reductive dechlorination in dense non-aqueous phase liquid source-zones. Adv Water Resour 30(6-7):1547-1561. https://doi.org/10.1016/j.advwatres. 2006.05.024

Colombani N, Pantano A, Mastrocicco M, Petitta M (2014) Reactive modelling of 1,2-DCA and DOC near the shoreline. J Contam Hydrol 169:100-111. https://doi.org/10.1016/j.jconhyd.2014.08. 003

Corapcioglu MY, Sung K, Kim J (2004) Parameter determination of sequential reductive dehalogenation reactions of chlorinated hydrocarbons. Transport Porous Med 55(2):169-182. https://doi.org/10. 1023/B:TIPM.0000010677.47179.f1

Cupples AM, Spormann AM, McCarty PL (2004) Vinyl chloride and cisdichloroethene dechlorination kinetics and microorganism growth 
under substrate limiting conditions. Environ Sci Technol 38(4): 1102-1107. https://doi.org/10.1021/es0348647

Da Silva ML, Alvarez P (2008) Exploring the correlation between halorespirer biomarker concentrations and TCE dechlorination rates. J Environ Eng 134(11):895-901. https://doi.org/10.1061/(ASCE) 0733-9372(2008)134:11(895)

Dillehay JL, Bowman KS, Yan J, Rainey FA, Moe WM (2014) Substrate interactions in dehalogenation of 1,2-dichloroethane, 1,2dichloropropane, and 1,1,2-trichloroethane mixtures by Dehalogenimonas spp. Biodegradation 25(2):301-312. https://doi. org/10.1007/s10532-013-9661-2

Duhamel M, Edwards EA (2006) Microbial composition of chlorinated ethene-degrading cultures dominated by Dehalococcoides. FEMS Microbiol Ecol 58(3):538-549. https://doi.org/10.1111/j.15746941.2006.00191.x

Duhamel M, Edwards EA (2007) Growth and yields of dechlorinators, acetogens, and methanogens during reductive dechlorination of chlorinated ethenes and dihaloelimination of 1,2-dichloroethane. Environ Sci Technol 41(7):2303-2310. https://doi.org/10.1021/ es062010r

Edwards EA (2014) Breathing the unbreathable. Science 346(6208):424425. https://doi.org/10.1126/science.1261194

Ellis DE, Lutz EJ, Odom JM, Buchanan RJ, Bartlett CL, Lee MD, Harkness MR, DeWeerd KA (2000) Bioaugmentation for accelerated in situ anaerobic bioremediation. Environ Sci Technol 34(11): 2254-2260. https://doi.org/10.1021/es990638e

EPA (2018) 2018 Edition of the drinking water standards and health advisories tables. US Environmental Protection Agency, Washington, DC, p 20

Field JA, Sierra-Alvarez R (2004) Biodegradability of chlorinated solvents and related chlorinated aliphatic compounds. Rev Environ Sci Biotechnol 3(3):185-254. https://doi.org/10.1007/s11157-0044733-8

Garant H, Lynd L (1998) Applicability of competitive and noncompetitive kinetics to the reductive dechlorination of chlorinated ethenes. Biotechnol Bioeng 57(6):751-755. https://doi.org/10.1002/(SICI) 1097-0290(19980320)57:6<751::AID-BIT13>3.0.CO;2-A

Grostern A, Edwards EA (2009) Characterization of a Dehalobacter coculture that dechlorinates 1,2-dichloroethane to ethene and identification of the putative reductive dehalogenase gene. Appl Environ Microbiol 75(9):2684-2693. https://doi.org/10.1128/AEM.0203708

Grostern A, Chan WW, Edwards EA (2009) 1,1,1-Trichloroethane and 1, 1-dichloroethane reductive dechlorination kinetics and cocontaminant effects in a Dehalobacter-containing mixed culture. Environ Sci Technol 43(17):6799-6807. https://doi.org/10.1021/ es901038x

Haest PJ, Springael D, Smolders E (2010a) Dechlorination kinetics of TCE at toxic TCE concentrations: assessment of different models. Water Res 44(1):331-339. https://doi.org/10.1016/j.watres.2009.09. 033

Haest PJ, Springael D, Smolders E (2010b) Modelling reactive CAH transport using batch experiment degradation kinetics. Water Res 44(9):2981-2989. https://doi.org/10.1016/j.watres.2010.02.031

Haston ZC, McCarty PL (1999) Chlorinated ethene half-velocity coefficients (Ks) for reductive dehalogenation. Environ Sci Technol 33(2): 223-226. https://doi.org/10.1021/es9805876

Kouznetsova I, Mao X, Robinson C, Barry DA, Gerhard JI, McCarty PL (2010) Biological reduction of chlorinated solvents: batch-scale geochemical modeling. Adv Water Resour 33(9):969-986. https://doi. org/10.1016/j.advwatres.2010.04.017

Lai Y, Becker JG (2013) Compounded effects of chlorinated ethene inhibition on ecological interactions and population abundance in a Dehalococcoides-Dehalobacter coculture. Environ Sci Technol 47(3):1518-1525. https://doi.org/10.1021/es3034582
Lineweaver H, Burk D (1934) The determination of enzyme dissociation constants. J Am Chem Soc 56(3):658-666

Low A, Zhao S, Rogers MJ, Zemb O, Lee M, He J, Manefield M (2019) Isolation, characterization and bioaugmentation of an acidotolerant 1,2-dichloroethane respiring Desulfitobacterium species from a low pH aquifer. FEMS Microbiol Ecol 95(5):fiz055. https://doi.org/10. 1093/femsec/fiz055

Mackay D, Shiu WY (1981) A critical review of Henry's law constants for chemicals of environmental interest. J Phys Chem Ref Data 10(4):1175-1199

Malaguerra F, Chambon JC, Bjerg PL, Scheutz C, Binning PJ (2011) Development and sensitivity analysis of a fully kinetic model of sequential reductive dechlorination in groundwater. Environ Sci Technol 45(19):8395-8402. https://doi.org/10.1021/es201270z

Maness AD, Bowman KS, Yan J, Rainey FA, Moe WM (2012) Dehalogenimonas spp. can reductively dehalogenate high concentrations of 1,2-dichloroethane, 1,2-dichloropropane, and 1,1,2trichloroethane. AMB Express 2(1):54. https://doi.org/10.1186/ 2191-0855-2-54

Martín-González L, Hatijah Mortan S, Rosell M, Parladé E, MartínezAlonso M, Gaju N, Caminal G, Adrian L, Marco-Urrea E (2015) Stable carbon isotope fractionation during 1,2-dichloropropane-topropene transformation by an enrichment culture containing Dehalogenimonas strains and a $d c p A$ gene. Environ Sci Technol 49(14):8666-8674. https://doi.org/10.1021/acs.est.5b00929

Marzorati M, De Ferra F, Van Raemdonck H, Borin S, Allifranchini E, Carpani G, Serbolisca L, Verstraete W, Boon N, Daffonchio D (2007) A novel reductive dehalogenase, identified in a contaminated groundwater enrichment culture and in Desulfitobacterium dichloroeliminans strain DCA1, is linked to dehalogenation of 1,2dichloroethane. Appl Environ Microbiol 73(9):2990-2999. https:// doi.org/10.1128/AEM.02748-06

Mayer-Blackwell K, Fincker M, Molenda O, Callahan B, Sewell H, Holmes S, Edwards EA, Spormann AM (2016) 1,2Dichloroethane exposure alters the population structure, metabolism, and kinetics of a trichloroethene-dechlorinating dehalococcoides mecartyi consortium. Environ Sci Technol 50(22):12187-12196. https://doi.org/10.1021/acs.est.6b02957

Maymó-Gatell X, Anguish T, Zinder SH (1999) Reductive dechlorination of chlorinated ethenes and 1,2-dichloroethane by "Dehalococcoides ethenogenes" 195. Appl Environ Microbiol 65(7):3108-3113

Meckenstock RU, Elsner M, Griebler C, Lueders T, Stumpp C, Aamand J, Agathos SN, Albrechtsen H-J, Bastiaens L, Bjerg PL (2015) Biodegradation: updating the concepts of control for microbial cleanup in contaminated aquifers. Environ Sci Technol 49(12): 7073-7081. https://doi.org/10.1021/acs.est.5b00715

Moe WM, Yan J, Nobre MF, da Costa MS, Rainey FA (2009) Dehalogenimonas lykanthroporepellens gen. nov., sp. nov., a reductively dehalogenating bacterium isolated from chlorinated solventcontaminated groundwater. Int J Syst Evol Microbiol 59(11):26922697. https://doi.org/10.1099/ijs.0.011502-0

Moe WM, Rainey FA, Yan J (2016) The genus Dehalogenimonas. In: Adrian L, Löffler FE (eds) Organohalide-respiring bacteria. Springer, Berlin, pp 137-151

Muyzer G, De Waal EC, Uitterlinden AG (1993) Profiling of complex microbial populations by denaturing gradient gel electrophoresis analysis of polymerase chain reaction-amplified genes coding for 16S rRNA. Appl Environ Microbiol 59(3):695-700

Parthasarathy A, Stich TA, Lohner ST, Lesnefsky A, Britt RD, Spormann AM (2015) Biochemical and EPR-spectroscopic investigation into heterologously expressed vinyl chloride reductive dehalogenase (VcrA) from Dehalococcoides mccartyi strain VS. J Am Chem Soc 137(10):3525-3532. https://doi.org/10.1021/ja511653d

Pérez-de-Mora A, Lacourt A, McMaster ML, Liang X, Dworatzek SM, Edwards EA (2018) Chlorinated electron acceptor abundance drives selection of Dehalococcoides mccartyi (D. mccartyi) strains in 
dechlorinating enrichment cultures and groundwater environments. Front Microbiol 9:812. https://doi.org/10.3389/Fmicb.2018.00812

Sander R (2015) Compilation of Henry's law constants (version 4.0) for water as solvent. Atmos Chem Phys 15(8):4399-4981. https://doi. org/10.5194/acp-15-4399-2015

Schaefer CE, Condee CW, Vainberg S, Steffan RJ (2009) Bioaugmentation for chlorinated ethenes using Dehalococcoides sp.: comparison between batch and column experiments. Chemosphere 75(2):141-148. https://doi.org/10.1016/j. chemosphere.2008.12.041

Schneidewind U, Haest PJ, Atashgahi S, Maphosa F, Hamonts K, Maesen M, Calderer M, Seuntjens P, Smidt H, Springael D, Dejonghe W (2014) Kinetics of dechlorination by Dehalococcoides mccartyi using different carbon sources. J Contam Hydrol 157:25-36. https://doi.org/10.1016/j.jconhyd.2013.10.006

Smits TH, Devenoges C, Szynalski K, Maillard J, Holliger C (2004) Development of a real-time PCR method for quantification of the three genera Dehalobacter, Dehalococcoides, and Desulfitobacterium in microbial communities. J Microbiol Methods 57(3):369-378. https://doi.org/10.1016/j.mimet.2004.02. 003

Stams AJ, Van Dijk JB, Dijkema C, Plugge CM (1993) Growth of syntrophic propionate-oxidizing bacteria with fumarate in the absence of methanogenic bacteria. Appl Environ Microbiol 59(4): 1114-1119

Staudinger J, Roberts PV (2001) A critical compilation of Henry's law constant temperature dependence relations for organic compounds in dilute aqueous solutions. Chemosphere 44(4):561-576. https:// doi.org/10.1016/S0045-6535(00)00505-1

Sung Y, Fletcher KE, Ritalahti KM, Apkarian RP, Ramos-Hernández N, Sanford RA, Mesbah NM, Löffler FE (2006) Geobacter lovleyi sp. nov. strain SZ, a novel metal-reducing and tetrachloroethenedechlorinating bacterium. Appl Environ Microbiol 72(4):27752782. https://doi.org/10.1128/AEM.72.4.2775-2782.2006

Sutton NB, Atashgahi S, Saccenti E, Grotenhuis T, Smidt H, Rijnaarts HH (2015) Microbial community response of an organohalide respiring enrichment culture to permanganate oxidation. PLoS One 10(8):e0134615. https://doi.org/10.1371/journal.pone.0134615

Vandermaesen J, Horemans B, Bers K, Vandermeeren P, Herrmann S, Sekhar A, Seuntjens P, Springael D (2016) Application of biodegradation in mitigating and remediating pesticide contamination of freshwater resources: state of the art and challenges for optimization.
Appl Microbiol Biotechnol 100(17):7361-7376. https://doi.org/10. 1007/s0025

Vrugt JA, Robinson BA (2007) Improved evolutionary optimization from genetically adaptive multimethod search. Proc Natl Acad Sci 104(3):708-711. https://doi.org/10.1073/pnas.0610471104

Wang S, He J (2013) Dechlorination of commercial PCBs and other multiple halogenated compounds by a sediment-free culture containing Dehalococcoides and Dehalobacter. Environ Sci Technol 47(18):10526-10534. https://doi.org/10.1021/es4017624

Weatherill JJ, Atashgahi S, Schneidewind U, Krause S, Ullah S, Cassidy N, Rivett MO (2018) Natural attenuation of chlorinated ethenes in hyporheic zones: a review of key biogeochemical processes and insitu transformation potential. Water Res 128:362-382. https://doi. org/10.1016/j.watres.2017.10.059

Werisch S, Grundmann J, Al-Dhuhli H, Algharibi E, Lennartz F (2014) Multiobjective parameter estimation of hydraulic properties for a sandy soil in Oman. Environ Earth Sci 72(12):4935-4956. https:// doi.org/10.1007/s12665-014-3537-6

Wöhling T, Vrugt JA, Barkle GF (2008) Comparison of three multiobjective optimization algorithms for inverse modeling of vadose zone hydraulic properties. Soil Sci Soc Am J 72(2):305-319. https://doi.org/10.2136/sssaj2007.0176

Yang Y, Higgins SA, Yan J, Şimşir B, Chourey K, Iyer R, Hettich RL, Baldwin B, Ogles DM, Löffler FE (2017) Grape pomace compost harbors organohalide-respiring Dehalogenimonas species with novel reductive dehalogenase genes. ISME J 11(12):2767-2780. https:// doi.org/10.1038/ismej.2017.127

Yu S, Semprini L (2004) Kinetics and modeling of reductive dechlorination at high PCE and TCE concentrations. Biotechnol Bioeng 88(4): 451-464. https://doi.org/10.1002/Bit.20260

Yu S, Dolan ME, Semprini L (2005) Kinetics and inhibition of reductive dechlorination of chlorinated ethylenes by two different mixed cultures. Environ Sci Technol 39(1):195-205. https://doi.org/10.1021/ es0496773

Yu R, Peethambaram HS, Falta RW, Verce MF, Henderson JK, Bagwell CE, Brigmon RL, Freedman DL (2013) Kinetics of 1,2-dichloroethane and 1,2-dibromoethane biodegradation in anaerobic enrichment cultures. Appl Environ Microbiol 79(4):1359-1367. https://doi.org/ 10.1128/AEM.02163-12

Publisher's note Springer Nature remains neutral with regard to jurisdictional claims in published maps and institutional affiliations. 